\title{
Expression of Pectate Lyase from Colletotrichum gloeosporioides in C. magna Promotes Pathogenicity
}

\author{
Nir Yakoby, ${ }^{1,3}$ Stanley Freeman, ${ }^{2}$ Amos Dinoor, ${ }^{3}$ Noel T. Keen, ${ }^{4}$ and Dov Prusky ${ }^{1}$ \\ ${ }^{1}$ Department of Postharvest Science of Fresh Produce and ${ }^{2}$ Department of Plant Pathology, Agricultural \\ Research Organization, The Volcani Center, Bet Dagan 50250, Israel; ${ }^{3}$ Department of Plant Pathology \\ and Microbiology, The Hebrew University of Jerusalem, Faculty of Agricultural, Food and Environmental \\ Quality Sciences, Rehovot 76100, Israel; ' ${ }^{2}$ epartment of Plant Pathology, University of California, \\ Riverside 95250, U.S.A. \\ Accepted 2 May 2000.
}

To test the contribution of pectate lyase (PL) to promoting fungal pathogenicity, a pectate lyase gene (pel) from the avocado pathogen Colletotrichum gloeosporioides, isolate Cg-14, was expressed in $C$. magna isolate $\mathrm{L}-2.5$, a pathogen of cucurbits that causes minor symptoms in watermelon seedlings and avocado fruits. Isolate L-2.5 was transformed with pPCPH-1 containing $h p h-B$ as a selectable marker and the 4.1-kb genomic pel clone. Southern hybridization, with the 4.1-kb genomic pel clone or 2.13-kb $h p h-B$ cassette as probes, detected integration of pel in transformed $C$. magna isolates Cm-PL-3 and CmPL-10. Western blot (immunoblot) analysis with antibodies against Cg-14 PL detected a single PL secreted by L-2.5 at a molecular mass of $41.5 \mathrm{kDa}$, whereas the PL of $C$. gloeosporioides had a molecular mass of $39 \mathrm{kDa}$. When PL activity was measured 4 days after inoculation in pectolytic enzyme-inducing media (PEIM), transformed isolates Cm-PL-3 and Cm-PL-10 showed additive PL activity relative to both $\mathrm{Cg}-14$ and $\mathrm{L}-2.5$. Transformed isolates also showed additive maceration capabilities on avocado pericarp relative to the wild-type $C$. magna alone, but did not reach the maceration ability of $C$. gloeosporioides. However, more severe maceration and damping off developed in watermelon seedlings inoculated with the transformed isolates compared with the two wild-type isolates, which showed no symptom development on these seedlings during the same period. Results clearly show the contribution of a single pel to the pathogenic abilities of $C$. magna and suggest that PL is a pathogenicity factor required for the penetration and colonization of Colletotrichum species.

Additional keywords: pectolytic enzymes.

Colletotrichum gloeosporioides, a pathogen of avocado fruits, has been found to produce endopolygalacturonase (endo-PG) (Prusky et al. 1989), pectin lyase (PNLA) (Bowen

Corresponding author: Dov Prusky; Fax: 972-3-968 3622; E-mail: prusky33@netvision.net.il

Nucleotide and/or amino acid sequence data can be found at the GenBank data base as accession no. AF052632. et al. 1995), pectin methyl esterase (PME) (Ortega 1996), and pectate lyase (PL) (Wattad et al. 1997) during the colonization of infected tissue. Evidence of the importance of PL secretion during Colletotrichum colonization on avocado fruits has been found in a number of studies: (i) reduced symptom development of a C. magna mutant (Path-1) with limited secretion of PL (Wattad et al. 1995); (ii) inhibition of decay following coinoculation of $C$. gloeosporioides spores with PL antibodies (Wattad et al. 1997); (iii) the activity inhibition of pectolytic enzymes by the host flavonoid epicatechin, which correlated with the inhibition of symptom development (Wattad et al. 1994); and (iv) a lack of decay development under conditions that are not permissive to PL secretion (Yakoby et al. 2000). These results suggest that PL is a limiting factor during the early stages of pathogenesis (Wattad et al. 1997; Yakoby et al. 2000).

Cell-wall-degrading enzymes (CWDEs) are considered to play a role in the pathogenesis of bacteria and fungi on their hosts (Collmer and Keen 1986; Ried and Collmer 1988; Annis and Goodwin 1997). A significant amount of work has been published on the role of pectolytic enzymes, but only a few reports have shown their clear involvement in fungal pathogenicity (Shieh et al. 1997; ten Have et al. 1998). Of the approaches that have been taken to demonstrate this involvement-(i) gene disruption; (ii) gene complementation of a deficient mutant; (iii) heterologous gene expression; and (iv) antisense gene expression - the first has been considered the most direct tool (Bowyer et al. 1995). However, when disrupted mutants are targeted in complex systems such as that of CWDEs, where several enzymes are secreted during fungal attack, the disruption of a single enzyme may be complemented by the activity of other secreted CWDEs (Hamer and Holden 1997). Indeed, most publications on disrupted CWDEs show no reduction in pathogenicity (i.e., Bowen et al. 1995; Gou et al. 1995; Sheng-Cheng et al. 1997). These reports suggest that the secretion of other CWDEs that share similar or overlapping enzymatic activities can easily overcome the loss of one enzyme. On the other hand, disruption of pecA, encoding the endo-PG $\mathrm{P} 2 \mathrm{c}$ in the saprophytic fungus Aspergillus flavus, resulted in decreased cotton ball invasion (Shieh et al. 1997). Furthermore, the disruption of Bcpg1, one 
of the genes encoding PG of Botrytis cinerea, reduced its colonization by 22 to $50 \%$ (ten Have et al. 1998).

Heterologous expression was used to test the role of PG (pg1) from Fusarium oxysporum f. sp. lycopersici in a pg1deficient isolate of $F$. oxysporum f. sp. melonis, where the PGexpressing isolate showed no change in virulence (Di Pietro and Roncero 1998). On the other hand, Dickman et al. (1989) showed that the insertion of a cutinase gene from $F$. oxysporum f. sp. pisi into species of Mycospherella, a pathogen that usually penetrates through wounds, enabled the latter's direct penetration through the cuticle. Heterologous expression was also used for complementation of the melanin biosynthetic pathway from Alternaria alternata in a deficient Magnaporthe grisea mutant, restoring the latter's pathogenicity (Kawamura et al. 1997).

To evaluate the contribution of a single enzyme to the pathogenic abilities of a fungus, we expressed a $4.1-\mathrm{kb}$ clone encoding PL from $C$. gloeosporioides in C. magna, a weak pathogen of avocado and watermelon that colonizes both hosts (Prusky et al. 1994). Comparison of the genomic clone pel (partial sequence $[1.52 \mathrm{~kb}]$ of the clone is published [accession number AF052632, GenBank]) to the pel cDNA clone (accession number U329242, GenBank) showed no introns in the latter. The sequence surrounding the ATG start codon, 5' CAAGATGAA $3^{\prime}$, closely resembles the Kozak sequence, 5' CAMMATGNC $3^{\prime}$, identified in the genes of filamentous fungi and higher eukaryotic genes (Ballance 1986; Kozak 1986).
A transformation vector was constructed by subcloning the 2.13-kb $h p h-B$ cassette (hygromycin phosphotransferase) from pHA-1.3 (Redman and Rodriguez 1994) into pGEM-7Z at sites $X b a \mathrm{I}$ and HindIII, generating pGH-1. The 4.1-kb pel clone, restricted with EcoRI, was blunt ended and subcloned into the plasmid $\mathrm{pGH}-1$ at the blunt-ended $\mathrm{XbaI}$ site, generating the $9.23-\mathrm{kb}$ pPCPH-1. Protoplasts of the wild-type $C$. magna isolate L- 2.5 were transformed with $1 \mu \mathrm{g}$ of pPCPH-1 and selected as previously described (Redman and Rodriguez 1994). Two transformed isolates, Cm-PL-3 and Cm-PL-10, from 20 colonies, were randomly selected for further analysis. One isolate, GH-2, transformed with pGH-1, was selected and further analyzed for the effect of hygromycin on virulence. The isolate was found to be hygromycin resistant, contained the $h p h-B$ cassette, and possessed the same virulence abilities as wild-type $C$. magna (data not shown).

Southern blot analysis of the transformed C. magna and two wild-type isolates, $\mathrm{Cg}$-14 and L-2.5, showed integration of the plasmid at different sites in the genome, when probed with the 4.1-kb pel clone (Fig. 1A) or with the 2.13-kb hph-B cassette (Fig. 1B). Western blot analysis of the proteins secreted from Cm-PL-3 and Cm-PL-10 into pectolytic enzyme-inducing media (PEIM; Prusky et al. 1989) revealed the presence of two bands of 39 and $41.5 \mathrm{kDa}$, corresponding to the PL bands in Cg-14 and L-2.5, respectively (Fig. 2).

PL activity of the proteins secreted into PEIM was measured as described by Collmer et al. (1988). Four days after
A

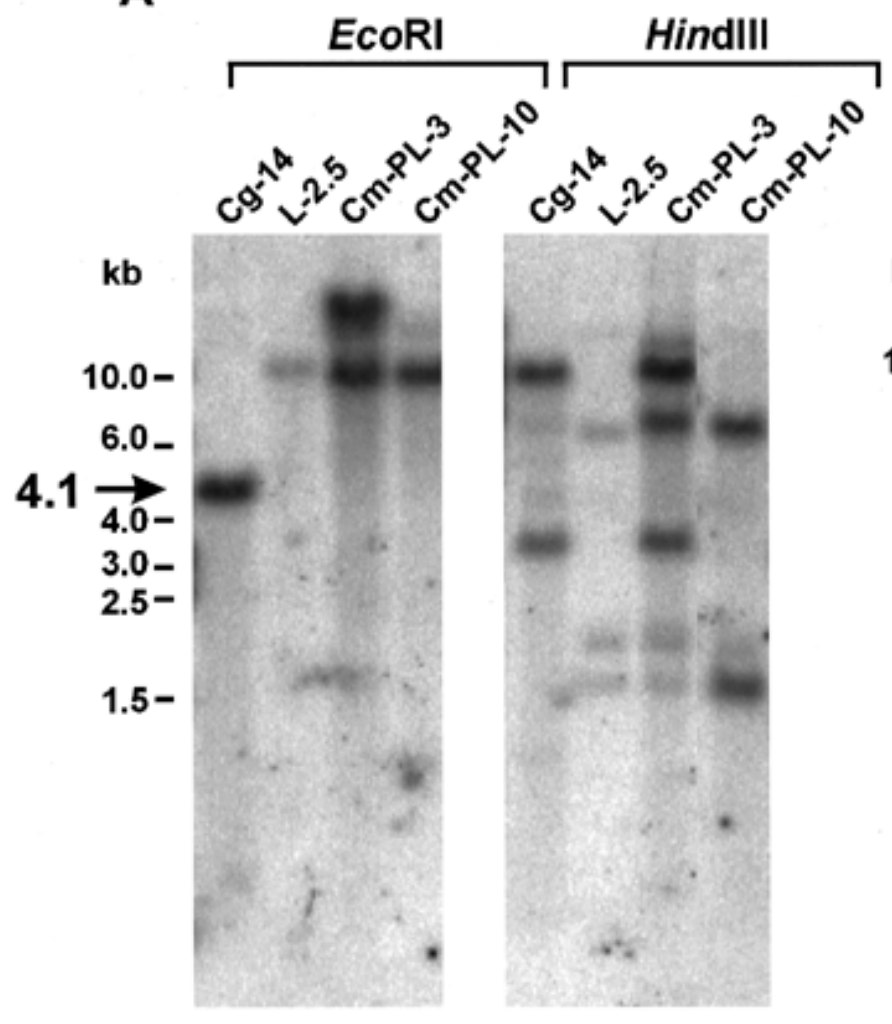

B

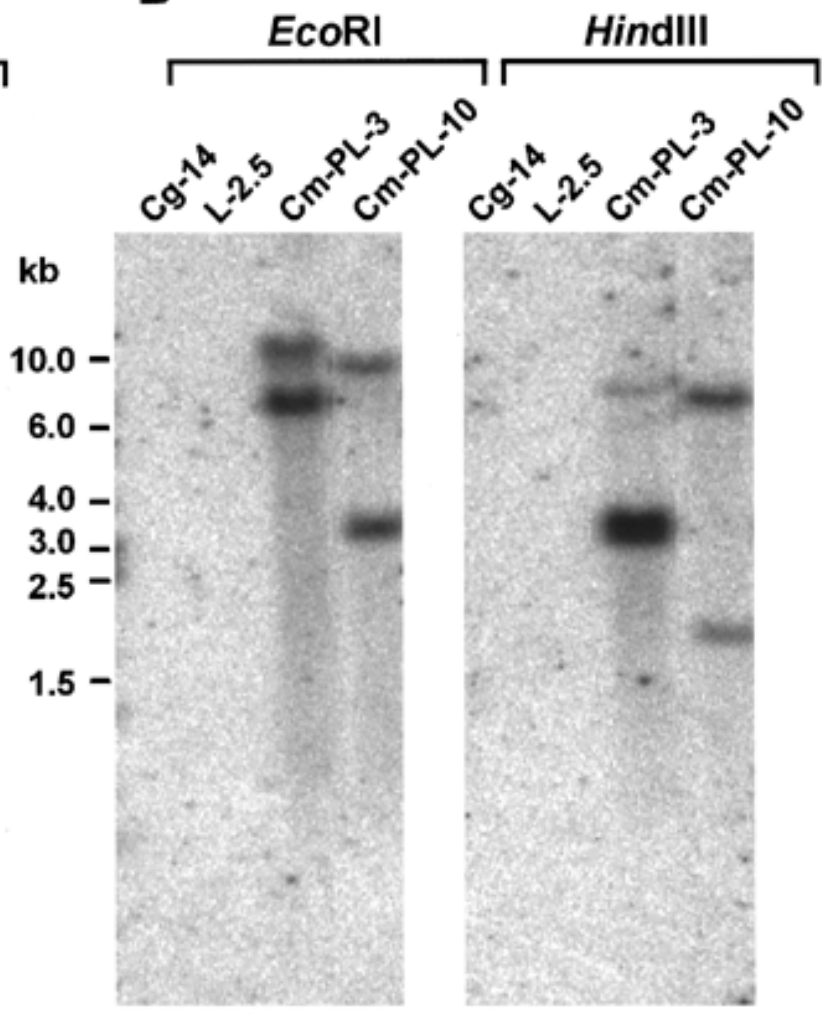

Fig. 1. Southern blot analysis of the transformed isolates, Cm-PL-3 and Cm-PL-10, compared with wild-type isolate Cg-14 of Colletotrichum gloeosporioides and wild-type isolate L-2.5 of C. magna. Fungal DNA was extracted as described by Rodriguez (1993) from $1 \mathrm{~g}$ of dry mycelium. Fungal DNA $(5 \mu \mathrm{g})$ was restricted for $15 \mathrm{~h}$ with $10 \mathrm{U}$ of EcoRI or HindIII restriction enzymes (Promega, Madison, WI). Restricted DNA was subjected to Southern blot (Sambrook et al. 1989) onto a HyBond ${ }^{+}$nylon membrane (Amersham, Buckinghamshire, UK). All hybridizations were carried out at $65^{\circ} \mathrm{C}$ and washes were with $0.1 \times \mathrm{SSC}(1 \times \mathrm{SSC}$ is $0.15 \mathrm{M} \mathrm{NaCl}$ plus $0.015 \mathrm{M}$ sodium citrate). Membrane was probed with (A) $4.1-\mathrm{kb}$ pel or (B) $2.13-\mathrm{kb}$ hph-B. 
isolate growth, PL activity was $3.1 \times 10^{-3} \mathrm{U} / \mathrm{min}$ for $C$. gloeosporioides, and $4.1 \times 10^{-3} \mathrm{U} / \mathrm{min}$ for C. magna. Increased activity, reaching 168 and $231 \%$ of the wild-type $C$. magna isolate L-2.5, was observed in Cm-PL-3 and Cm-PL10, respectively. Activity was calculated from each isolate's regression line formula $\left(r^{2}=0.999\right.$, for all four isolates). All

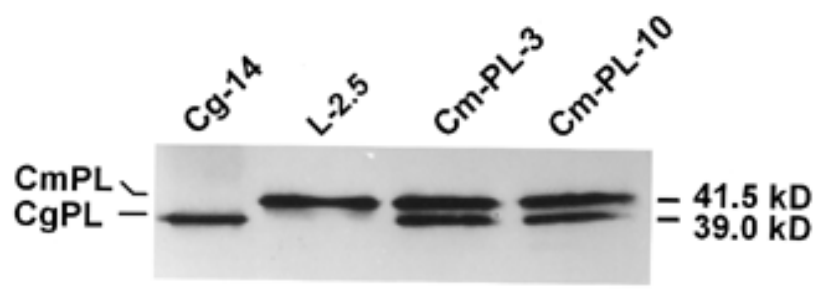

Fig. 2. Pectate lyase (PL) secretion from the wild-type isolates $\mathrm{Cg}-14$ and L-2.5, and the transformed isolates, Cm-PL-3 and Cm-PL-10. Isolates were grown for 4 days in pectolytic enzyme-inducing media (PEIM). Clear supernatant $(100 \mathrm{ml})$ was concentrated by rotavapor (Buchii, Flawil, Switzerland) at $42^{\circ} \mathrm{C}$ and samples $(10 \mu \mathrm{l})$ were subjected to Western blot (immunoblot) analysis by running on a $12.5 \%$ SDS (sodium dodecyl sulfate)-polyacrylamide gel (Mini-Protean II, BioRad Laboratories, Hercules, CA) for $1 \mathrm{~h}$ at $150 \mathrm{~V}$ constant voltage and transferring to a nitrocellulose membrane (Pall Gelman Sciences, Northampton, UK), which was analyzed with PL antibodies as described by Wattad et al. (1995).

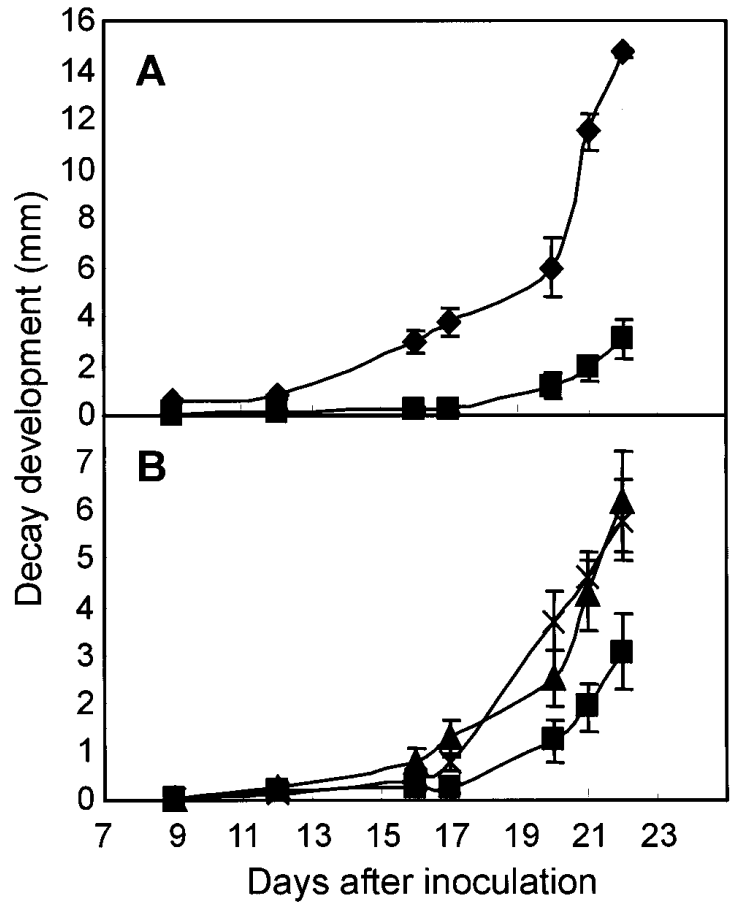

Fig. 3. Decay development on avocado fruit cv. Fuerte. Freshly harvested avocado fruits (20 fruits) were inoculated with (A) a 10- $\mu$ l conidia suspension $\left(1 \times 10^{6}\right.$ conidia per $\left.\mathrm{ml}\right)$ of $\mathrm{Cg}-14(\bullet)$ or L-2.5 ( $\left.\mathbf{\square}\right)$, or with (B) L-2.5 (ם), Cm-PL-3 ( $\mathbf{\Delta}$ ) or Cm-PL-10 (X). Isolates were spotinoculated twice and compared with $\mathrm{Cg}-14$ inoculation on the opposite side of the same fruit. Decay diameter was monitored during fruit storage at $20^{\circ} \mathrm{C}$. Results show one representative experiment out of three independent experiments and the standard error.
PL activities were significantly different $(P<0.01)$ between the different isolates. PL activity was additive for the transformed isolate Cm-PL-3 and significantly more than additive for Cm-PL-10, relative to the two wild-type isolates $\mathrm{Cg}-14$ and L-2.5. Although both isolates were transformed with the same gene, PL activity was significantly greater for transformed isolate Cm-PL-10 than for Cm-PL-3. The differences in activities may be explained by the different patterns of gene integration into the fungal genome, observed by Southern blot analysis, which may have affected the expression of the integrated pel, as has been described for higher plants and fungi (MacKenzie et al. 1993).

Germinated conidia of wild-type $C$. magna isolate L-2.5 produced appressoria that breached the cuticle of the avocado fruit (cv. Fuerte) but caused only a 2-mm-diameter lesion after 5 days (Fig. 3A). Decay caused by $\mathrm{Cg}-14$ was fourfold larger in diameter than that caused by L-2.5 (Fig. 3A). Transformed isolates Cm-PL-3 and Cm-PL-10 showed a 1.8-fold increase in decay, compared with L-2.5 (Fig. 3B). Inoculation of avocado mesocarp with the different isolates showed no difference in maceration development (data not presented). The fact that the transformed isolates were more aggressive on avocado pericarp, but that these differences were eliminated when inoculation was carried out in the avocado mesocarp, further

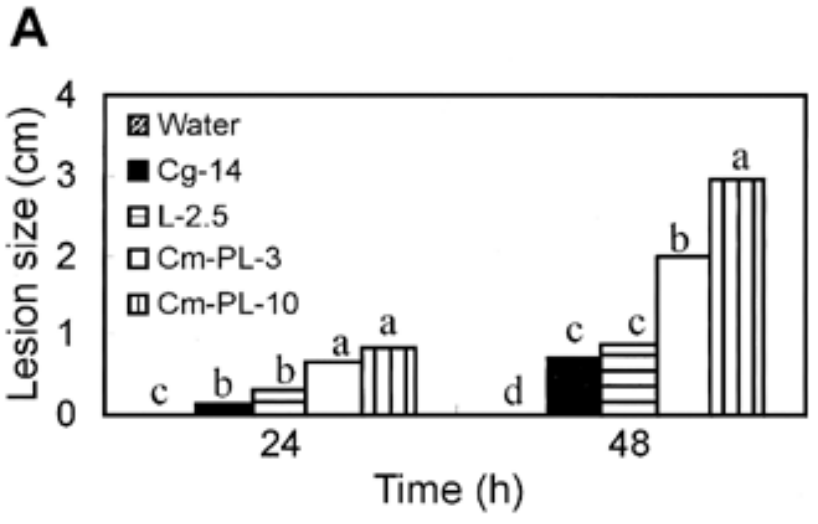

B

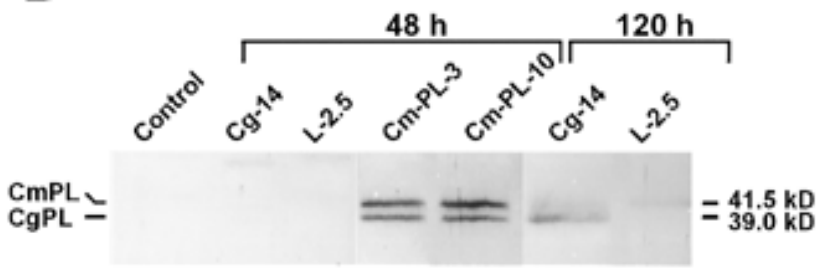

Fig. 4. Lesion development on detached watermelon seedling stems (cv. Odem) formed by Cg-14, L-2.5, Cm-PL-3, Cm-PL-10, or water. Detached, 10-day-old watermelon (cv. Odem) seedling stems were wounded by pin-pricking and inoculated with $5 \mu \mathrm{l}$ per stem of each of the different isolates at $4 \times 10^{6}$ conidia per ml. A, Extent of tissue colonization was measured 24 and $48 \mathrm{~h}$ post inoculation. B, Pectate lyase (PL) from inoculated stem tissue 48 and $120 \mathrm{~h}$ post inoculation was detected on eight inoculated stems per isolate, and a water control. Stems were maintained under fluorescent light at $20^{\circ} \mathrm{C}$ in $90 \%$ humidity for $120 \mathrm{~h}$. Samples were homogenized when the whole $5-\mathrm{cm}$ stem sections were macerated and $10 \mu \mathrm{g}$ of protein was loaded for Western blot (immunoblot) analysis. All experiments were conducted three times. A, Letters above bars represent significant differences between isolates $(P<$ 0.01 ) according to analysis of variance. 


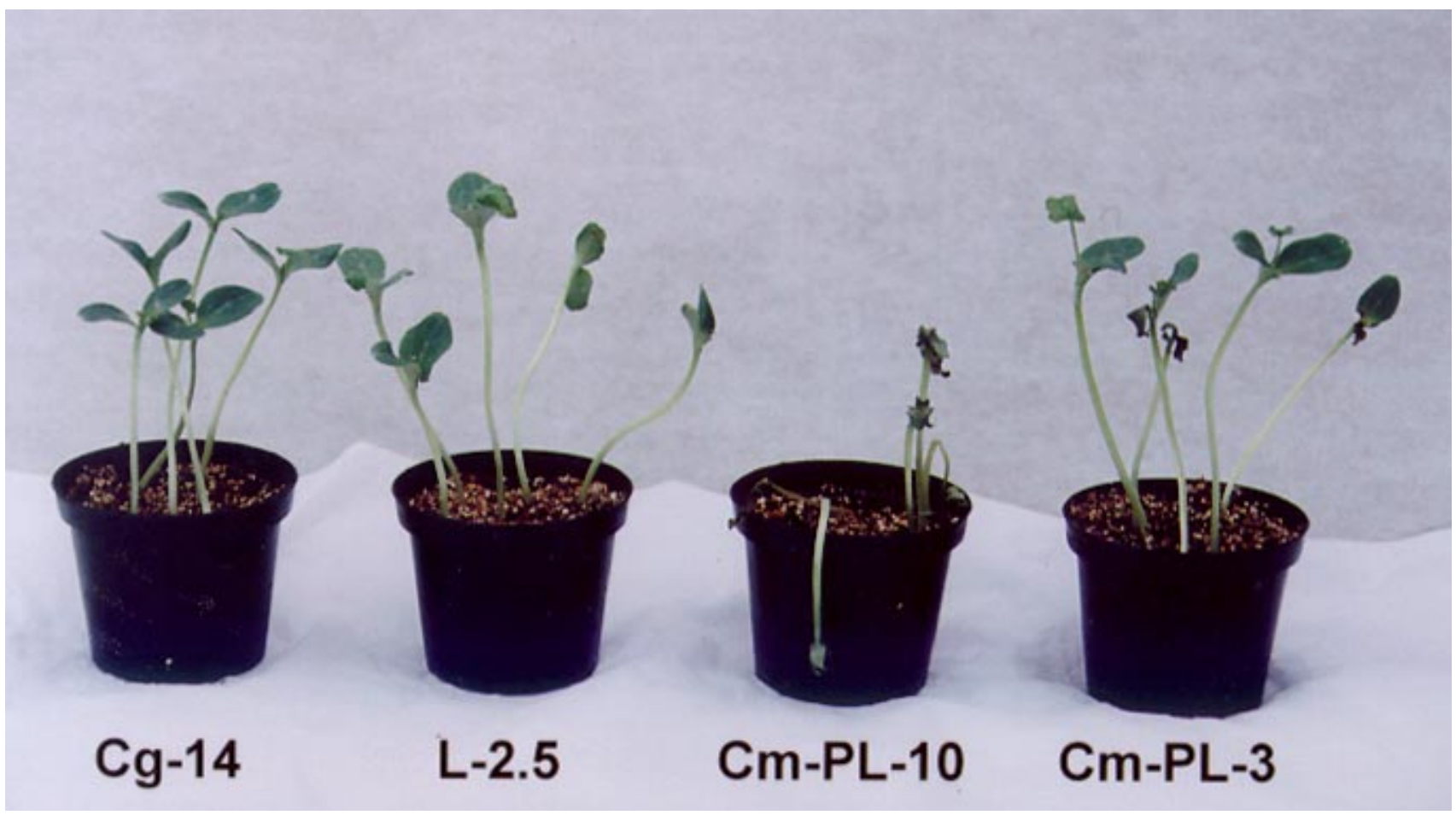

Fig. 5. Symptoms of necrotic development and damping off on watermelon seedlings (cv. Odem) caused by Cg-14, L-2.5, Cm-PL-3, or Cm-PL-10. Tenday-old watermelon seedlings were inoculated on the apex with $20 \mu \mathrm{l}$ of conidial suspension at a concentration of $4 \times 10^{6}$ conidia per ml of each different isolate. Plants were maintained in a greenhouse at $25^{\circ} \mathrm{C}$ in $90 \%$ humidity for $48 \mathrm{~h}$. Water inoculation was used as a control. Pathogenicity was observed 7 days after inoculation as developing necrosis and damping off.

supports the importance of PL for the initial stages of fungal host colonization.

When detached watermelon seedling stems were inoculated, both transformed isolates caused significantly larger lesion development 24 and $48 \mathrm{~h}$ post inoculation (Fig. 4A). PL secretion during the colonization of watermelon seedling stems by Cm-PL-3 and Cm-PL-10 was detected $48 \mathrm{~h}$ post inoculation, for Cg-14 and L-2.5 only after $120 \mathrm{~h}$ (Fig. 4B). Inoculation of watermelon seedlings with Cm-PL-10 and Cm-PL-3 caused damping off and leaf necrosis, whereas no symptoms developed with the two wild-type isolates Cg-14 and L-2.5, 6 days post inoculation (Fig. 5). The initial symptoms on the seedlings were soft maceration, indicating the activity of pectin-degrading enzymes. The increase in PL activity accompanied by the detection of both PLs in the tissue $48 \mathrm{~h}$ after inoculation, in contrast to $120 \mathrm{~h}$ in the two wild-type isolates, suggests the involvement of PL in tissue maceration. Since $\mathrm{Cg}$-14 secretes PL but causes much-reduced symptoms on detached watermelon, we assume that the combination of the transformed isolate with the new introduced PL increased its aggressiveness on the watermelon host. Various explanations may be considered as a basis for increased aggressiveness: the expressed PL promotes improved penetration of the stem vessels (Freeman and Rodriguez 1993) and during colonization enables more efficient expression of other virulence factors. It may also be that PL levels above the basic threshold of L-2.5 enable enhanced colonization of the plant tissue. It is clear from symptom development that the introduced PL affects the basic compatibility response of the watermelon seedlings.

Until now, most reports on the disruption of a single CWDE or combinations of CWDEs have shown limited or no contri- bution to the pathogenic abilities of the pathogen; for example, disruption of PNLA from C. gloeosporioides and PL from Nectria haematococca did not reduce their virulence (Bowen et al. 1995; Gou et al. 1995). Mutations in all known pel genes of Erwinia chrysanthemi reduced its virulence but the bacterium was still able to macerate the tissue (Ried and Collmer 1988), suggesting that a second set of PLs were activated (Kelemu and Collmer 1993). Gene disruption of the two endo$\beta$-1,4-xylanases from $M$. grisea had no effect on pathogenicity and revealed the secretion of an additional xylanase enzyme (Sheng-Cheng et al. 1997).

Our system further elucidates, by heterologous expression, the importance of PL as a pathogenicity and virulence factor (penetration and colonization) in watermelon seedlings and as a virulence factor (colonization) in avocado fruits. The addition of PL with its native control system demonstrated differences in attack between hosts and on the same host depending on the assay. This supports heterologous expression as a tool for determining the importance of specific genes in pathogenicity, and at the same time demonstrates the complexity of a system that requires more than one factor to express the pronounced contribution of a single enzyme to fungal pathogenicity.

\section{ACKNOWLEDGMENTS}

This research was supported by Research Grants IS-2686-96 to D. P. and IS-250-95 to S. F. from BARD, The United States-Binational Agricultural Research and Development Fund, the German-Israel Agricultural Research Agreement (GIARA) for the Benefit of the Third World, and the U.S.-Israel Cooperative Development Research (CDR) Program. N. Y. was granted personal scholarships from the Israel Fruit Market- 
ing Board/Ministry of Agriculture \& Rural Development and from The Association of Trustees and Friends of the Agricultural Research Organization.

\section{LITERATURE CITED}

Annis, L. S., and Goodwin, H. P. 1997. Recent advances in the molecular genetics of plant cell wall-degrading enzymes produced by plant pathogenic fungi. Eur. J. Plant Pathol. 103:1-14.

Ballance, D. J. 1986. Sequences important in gene expression in filamentous fungi. Yeast 2:224-236.

Bowen, K. J., Templeton, D. M., Sharrock, R. K., Crowhurst, N. R., and Rikkerink, A. H. E. 1995. Gene inactivation in the plant pathogen Glomerella cingulata: Three strategies for the disruption of the pectin lyase gene pnlA. Mol. Gen. Genet. 246:196-205.

Bowyer, P., Clarke, B. R., Lunness, P., Daniels, M. J., and Osbourn, A. E. 1995. Host range of a plant pathogenic fungus determined by a saponin detoxification enzyme. Science 267:371-374.

Collmer, A., and Keen, T. N. 1986. The role of pectic enzymes in plant pathogenesis. Annu. Rev. Phytopathol. 24:383-409.

Collmer, A., Ried, L. J., and Mount, S. M. 1988. Assay methods for pectic enzymes. Methods Enzymol. 161:329-335.

Dickman, M. B., Podila, G. K., and Kolattukudy, P. E. 1989. Insertion of cutinase gene into a wound pathogen enables it to infect intact fruits. Nature 342:446-448.

Di Pietro, A., and Roncero, M. I. G. 1998. Cloning, expression, and role in pathogenicity of $p g 1$ encoding the major extracellular endopolygalacturonase of the vascular wilt pathogen Fusarium oxysporum. Mol. Plant-Microbe Interact. 11:91-98.

Freeman, S., and Rodriguez, R. J. 1993. Genetic conversion of a fungal plant pathogen to a nonpathogenic, endophytic mutalist. Science 260: 75-78.

Gou, W., Gonzalez-Candelas, L., and Kolattukudy, E. P. 1995. Cloning of a novel pectate lyase gene pelB from Fusarium solani f. sp. pisi (Nectria haematococca, mating type VI) and characterization of the gene product expressed in Pichia pastoris. J. Bacteriol. 177:7070-7077.

Hamer, J. E., and Holden, D. W. 1997. Linking approaches in the study of fungal pathogenesis: a commentary. Fungal Genet. Biol. 21:11-16.

Kawamura, C., Moriwaki, J., Kimura, N., Fujita, Y., Fuji, S., Hirano, T., Koizumi, S., and Tsuge, T. 1997. The melanin biosynthesis genes of Alternaria alternata can restore pathogenicity of the melanindeficient mutants of Magnaporthe grisea. Mol. Plant-Microbe Interact. 10:446-453.

Kelemu, S., and Collmer, A. 1993. Erwinia chrysanthemi EC16 produces a second set of plant inducible pectate lyase isozymes. Appl. Environ. Microbiol. 59:1756-1761.

Kozak, M. 1986. Point mutations close to the AUG initiator codon affect the efficiency of translation of rat preproinsulin in vivo. Nature 308: 241-247.

MacKenzie, D. A., Jeenes, J. D., Belshaw, N. J., and Archer, D. B. 1993.
Regulation of secreted protein production by filamentous fungi: recent developments and perspectives. J. Gen. Microbiol. 139:22952307.

Ortega, J. 1996. Pectolytic enzymes produced by the phytopathogenic fungus Colletotrichum gloeosporioides. Tex. J. Sci. 48:123-128.

Prusky, D., Freeman, S., Rodriguez, R. J., and Keen, N. T. 1994. A nonpathogenic mutant strain of Colletotrichum magna induces resistance to $C$. gloeosporioides in avocado fruits. Mol. Plant-Microbe Interact. 7:326-333.

Prusky, D., Gold, S., and Keen, N. T. 1989. Purification and characterization of an endopolygalacturonase produced by Colletotrichum gloeosporioides. Physiol. Mol. Plant Pathol. 35:121-133.

Redman, S. R., and Rodriguez, J. R. 1994. Factors affecting the efficient transformation of Colletotrichum species. Exp. Mycol. 18:230-246.

Ried, J. L., and Collmer, A. 1988. Construction and characterization of an Erwinia chrysanthemi mutant with directed deletions in all of the pectate lyase structural genes. Mol. Plant-Microbe Interact. 1:32-38.

Rodriguez, J. R. 1993. Polyphosphate present in DNA preparations from filamentous fungal species of Colletotrichum inhibits restriction endonucleases and other enzymes. Anal. Biochem. 209:291-297.

Sambrook, J., Fritsch, E. F., and Maniatis, T. A. 1989. Molecular Cloning: A Laboratory Manual. 2nd ed. Cold Spring Harbor Laboratory, Cold Spring Harbor, NY.

Sheng-Cheng, W., Kyung-Sik, H., Darvill, G. A., and Albersheim, P. 1997. Deletion of the two endo- $\beta-1,4$-xylanase genes reveals additional isozymes secreted by the rice blast fungus. Mol. Plant-Microbe Interact. 10:700-708.

Shieh, M., Brown, R. L., Whitehead, M. P., Carey, J. W., Cotty, P. J., Cleveland, T. E., and Dean, R. A. 1997. Molecular genetic evidence for the involvement of a specific polygalacturonase, $\mathrm{P} 2 \mathrm{c}$, in the invasion of Aspergillus flavus in cotton balls. Appl. Environ. Microbiol. 63:3548-3552.

ten Have, A., Mulder, W., Visser, J., and van Kan, J. A. L. 1998. The endopolygalacturonase gene $B \operatorname{cpg} 1$ is required for full virulence of Botrytis cinerea. Mol. Plant-Microbe Interact. 11:1009-1016.

Wattad, C., Dinoor, A., and Prusky, D. 1994. Purification of pectate lyase produced by Colletotrichum gloeosporioides and its inhibition by epicatechin: A possible factor involved in the resistance of unripe avocado fruits to anthracnose. Mol. Plant-Microbe Interact. 7:293297

Wattad, C., Freeman, S., Dinoor, A., and Prusky, D. 1995. A nonpathogenic mutant of Colletotrichum magna is deficient in extracellular secretion of pectate lyase. Mol. Plant-Microbe Interact. 8:621-626.

Wattad, C., Kobiler, D., Dinoor, A., and Prusky, D. 1997. Pectate lyase of Colletotrichum gloeosporioides attacking avocado fruits: cDNA cloning and involvement in pathogenicity. Physiol. Mol. Pathol. 50: 197-212.

Yakoby, N., Kobiler, I., Dinoor, A., and Prusky, D. 2000. pH regulation of pectate lyase secretion modulates the attack of Colletotrichum gloeosporioides on avocado fruits. Appl. Environ. Microbiol. 66: 1026-1030. 